Note

\title{
Formation Ratios of Zearalanone, Zearalenols, and Zearalanols versus Zearalenone during Incubation of Fusarium semitectum on Sorghum and Ratios in Naturally Contaminated Sorghum
}

\author{
Koji Aoyama $^{1, *}$, Eiichi Ishikuro ${ }^{2}$, Hiroko Noriduki ${ }^{3}$ and Masakatsu Ichinoe ${ }^{3,4}$ \\ ${ }^{1}$ Food and Agricultural Materials Inspection Center: 2-1 Shintoshin, Chuo-ku, Saitama 330-9731, Japan; \\ ${ }^{2}$ Japan Food Research Laboratories: 52-1 Motoyoyogi-cho, Shibuya-ku, Tokyo 151-0062, Japan; \\ ${ }^{3}$ Japan Grain Inspection Association: 1-2-1 Shiohama, Koto-ku, Tokyo 135-0043, Japan; \\ ${ }^{4}$ Professor Emeritus, Tokyo Kasei University: 1-18-1 Kaga, Itabashi-ku, Tokyo 173-8602, Japan; \\ ${ }^{*}$ Corresponding author
}

We incubated Fusarium semitectum on sorghum and measured the production of zearalenone (ZEN) and ZEN-related compounds (zearalanone (ZAN), $\alpha$-zearalenol ( $\alpha$-ZEL), $\beta$-zearalenol $(\beta$ ZEL), $\alpha$-zearalanol ( $\alpha$-ZAL) and $\beta$-zearalanol $(\beta$-ZAL)) in the culture by LC-MS. Of the five ZENrelated compounds, ZAN and $\beta$-ZEL were mainly detected. The concentrations of ZEN and the five ZEN-related compounds increased until 9 days after incubation and then increased slightly or stayed constant between days 9 and 15 . The ratios of $\alpha$-ZEL, $\beta$-ZEL, $\alpha$-ZAL and $\beta$-ZAL to ZEN decreased in a similar manner after 7 days, whereas the ratio of ZAN to ZEN remained constant after 5 days. Analysis of naturally contaminated sorghum by LC-MS/MS revealed that the production ratio of $\alpha$-ZEL to ZEN was inconsistent with that of our in vitro incubation analysis. The results indicate that ZAN might not be suitable for use as an internal standard.

(Received May 21, 2015)

Key words: Fusarium semitectum; sorghum; zearalanol; zearalanone; zearalenol; zearalenone

\section{Introduction}

Zearalenone (ZEN), a mycotoxin produced by Fusarium fungi, contaminates maize, barley, oat, sorghum (milo), and other cereal grains worldwide ${ }^{1)-3)}$. Although ZEN has relatively low acute toxicity, it can exert estrogenic effects on the reproductive system, particularly in swine $^{1)}$. Several compounds related to ZEN have been identified: zearalanone (ZAN), $\alpha$-zearalenol ( $\alpha$-ZEL), $\beta$ zearalenol ( $\beta$-ZEL), $\alpha$-zearalanol ( $\alpha$-ZAL, zeranol) and $\beta$-zearalanol $(\beta \text {-ZAL, taleranol) })^{3)}$ (Fig. 1). These ZENrelated compounds (ZRCs), except for $\beta$-ZEL, have stronger estrogenic activity than $\mathrm{ZEN}^{4-6)}$. In particular, the estrogenicity of $\alpha$-ZEL is 92 times greater than that of ZEN, as estimated by an improved MCF7 human breast cancer cell proliferation assay ${ }^{6}$.

The above-mentioned five ZRCs have been detected in urine, feces, and tissues ${ }^{3), 7)}$, as well as in processed foods ${ }^{8), 9)}$. In beer, $\beta$-ZEL, $\alpha$-ZEL and ZAN are formed from ZEN by the Saccharomyces cerevisiae strains used in brewing ${ }^{10)}$. ZEL and ZAL were also detected in Fusarium cultures ${ }^{4), 11), 12)}$. Therefore, these compounds are considered natural contaminants in grains; however, they commonly occur at lower concentrations than

\footnotetext{
* Corresponding author: kouji_aoyama@nm.famic.go.jp
}

$\mathrm{ZEN}^{11), 13)-17)}$. JECFA set a provisional maximum tolerable daily intake of ZEN at $0.5 \mu \mathrm{g} / \mathrm{kg}$ of body weight per day and recommends that the total intake of ZEN and its metabolites should not exceed this limit ${ }^{18)}$. However, little is known about the production of the $5 \mathrm{ZRCs}$ during incubation of Fusarium or about their relative concentrations in grains.

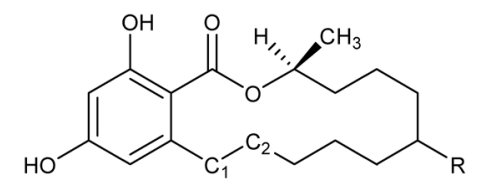

\begin{tabular}{lcccc}
\hline & Abbreviation & $\mathrm{C}_{1}-\mathrm{C}_{2}$ & $\mathrm{R}$ & $\begin{array}{c}\text { Relative } \\
\text { estrogenicity }\end{array}$ \\
\hline Zearalenone & $\mathrm{ZEN}$ & $\mathrm{C}=\mathrm{C}$ & $=\mathrm{O}$ & 1 \\
$\alpha$-Zearalenol & $\alpha-Z E L$ & $\mathrm{C}=\mathrm{C}$ & $\cdots \mathrm{OH}$ & 92 \\
$\beta$-Zearalenol & $\beta$-ZEL & $\mathrm{C}=\mathrm{C}$ & $-\mathrm{OH}$ & 0.44 \\
Zearalanone & $\mathrm{ZAN}$ & $\mathrm{C}-\mathrm{C}$ & $=\mathrm{O}$ & 2.5 \\
$\alpha$-Zearalanol & $\alpha-\mathrm{ZAL}$ & $\mathrm{C}-\mathrm{C}$ & $\cdots \mathrm{OH}$ & 18 \\
$\beta$-Zearalanol & $\beta$-ZAL & $\mathrm{C}-\mathrm{C}$ & $-\mathrm{OH}$ & 3.5 \\
\hline
\end{tabular}

Fig. 1. Chemical structures and relative estrogenicity values of ZEN and the 5 ZRCs

The relative estrogenicity of each compound was reported by Shier et al. ${ }^{6)}$ 
Sorghum is produced in areas where maize is not suitable for cultivation due to the hot or dry climate ${ }^{19)}$. Sorghum is one of the most important grains in the world, along with maize, rice, wheat and barley ${ }^{19)}$. Next to maize, sorghum is the main feed ingredient in Japan. We previously reported on ZEN contamination of sorghum $^{20)}$, and demonstrated that sorghum is a significant source of ZEN contamination, of which the primary causative fungus is $F$. semitectum. In this study, we examined the production of the 5 ZRCs during incubation of $F$. semitectum on sorghum and also measured their levels in naturally contaminated sorghums. This study is first report on in vitro production of ZRCs by $F$. semitectum. Although ZAN has been widely used as an internal standard, our results indicate that a different ZENrelated compound might be superior.

\section{Materials and Methods}

\section{Standards and reagents}

The standards of ZEN and the 5 ZRCs were purchased from Sigma-Aldrich (St. Louis, MO, USA). Stock solutions were prepared at a concentration of $100 \mu \mathrm{g} / \mathrm{mL}$ $(\beta$-ZEL) or $50 \mu \mathrm{g} / \mathrm{mL}$ (the others) in acetonitrile and stored at $-20^{\circ} \mathrm{C}$. The solvents used for LC-MS and LCMS/MS were of LC grade. Ammonium acetate ( $1 \mathrm{~mol} / \mathrm{L})$ solution was used for LC, and was purchased from Wako Pure Chemical Industries, Ltd. (Osaka, Japan). All other reagents used were of analytical grade.

\section{Fungal strain studied}

F. semitectum was isolated from sorghum as previously described ${ }^{20)}$. Briefly, ground sorghum was homogenized in $0.1 \%$ peptone-water. The diluted solution was placed on a dichloran rose bengal chloramphenicol agar (Oxoid, Cambridge, UK) plate, and incubated at $25^{\circ} \mathrm{C}$ for 5 to 7 days. The $F$. semitectum isolates were purified by subculturing on potato-dextrose agar slants and carnation leaf agar, and were identified based on macroscopic and microscopic characteristics.

\section{Determination of mycotoxin concentration in culture}

Unground sorghum and rice were obtained commercially, and used as artificial inoculation substrates. For production of ZEN and the $5 \mathrm{ZRCs}, F$. semitectum was inoculated on $30 \mathrm{~g}$ of sterilized substrate and incubated at $25^{\circ} \mathrm{C}$ for 14 days. To monitor the time courses of production of $\mathrm{ZEN}$ and the $5 \mathrm{ZRCs}$, the fungi inoculated on the sorghum substrate were incubated at $25^{\circ} \mathrm{C}$ for $3,5,7$, 9, 11, 13 and 15 days. The time course analysis was conducted in triplicate for days 3 to 11 and in quintuplicate for days 13 and 15 .

ZEN and the 5 ZRCs were extracted and purified according to the ZEN production assay for Fusarium species as previously described ${ }^{20)}$. In summary, ZEN and the 5 ZRCs were extracted by blending $25 \mathrm{~g}$ of the culture material with $100 \mathrm{~mL}$ of acetonitrile-water $(21: 4)$ for $5 \mathrm{~min}$. The extract was loaded onto a multifunctional clean-up column (MultiSep 226 AflaZon+, Romer Labs, Union, MO, USA). The first $4 \mathrm{~mL}$ of eluate was discard- ed, and $2 \mathrm{~mL}$ of the subsequent $3 \mathrm{~mL}$ was evaporated to dryness. The residue was redissolved in $1 \mathrm{~mL}$ of acetonitrile-water $(1: 1)$. The concentrations of ZEN and the 5 ZRCs in the purified solution were determined by LCMS with an atmospheric pressure chemical ionization (APCI) interface according to the conditions of our previous report ${ }^{20)}$ with the addition of monitoring of $5 \mathrm{ZRC}$ ions $(m / z 319$ for ZAN and $\alpha$ - or $\beta$-ZEL, and 321 for $\alpha$ - or $\beta$-ZAL). Detection of ZEN or the 5 ZRCs by LC-MS was confirmed by LC-MS/MS with an electrospray ionization (ESI) interface. The LC-MS/MS conditions used were as previously described ${ }^{20)}$. However, the following conditions were added in the present study: the collision energy for the $5 \mathrm{ZRCs}$ was set to $20 \mathrm{eV}$, and the precursor and product ions were selected to be $\mathrm{m} / z 319$ and 275, respectively, for ZAN and $\alpha$ - or $\beta$-ZEL, and 321 and 277, respectively, for $\alpha$ - or $\beta$-ZAL.

\section{Determination of mycotoxin concentrations in naturally} contaminated sorghum

Sorghum samples for detection of naturally occurring ZEN and the 5 ZRCs were collected in quantities of about $5 \mathrm{~kg}$ at a silo. All five samples were imported, three from Argentina, and the others from the United States. These samples were ground to particles less than $1 \mathrm{~mm}$ in diameter using an ultracentrifugal mill (ZM1, Retsch, Haan, Germany) in our laboratory and stored at $5^{\circ} \mathrm{C}$ prior to analysis.

ZEN and the 5 ZRCs in sorghum were detected using an official method for feed analysis in Japan*1 as follows: ZEN and the 5 ZRCs were extracted by shaking $50 \mathrm{~g}$ of the sample in a $300 \mathrm{~mL}$ flask with $100 \mathrm{~mL}$ of acetonitrile-water $(21: 4)$ for $60 \mathrm{~min}$. The extract was centrifuged at $650 \times \mathrm{g}$ for $5 \mathrm{~min}$ and the supernatant was loaded onto a MultiSep 226 AflaZon + . The initial $4 \mathrm{~mL}$ of eluate was discarded, and the following $2 \mathrm{~mL}$ was collected. A portion of the collected solution was mixed with the same amount of water-acetic acid (100:1). The solution was ultracentrifuged at $5,000 \times g$ for $5 \mathrm{~min}$, and the supernatant was analyzed by LC-MS/MS. Some LC-MS/MS conditions were different from those used to determine the concentration in culture. LC separation was performed using an Acquity UPLC system (Waters, Milford, MA, USA), and mass detection was performed using a Xevo TQD triple quadrupole mass spectrometer (Waters) equipped with ESI. A $10 \mu \mathrm{L}$ aliquot of sample was injected into and separated on a ZORBAX Eclipse XDB-C18 column $(150 \times 4.6 \mathrm{~mm}$ i.d., $5 \mu \mathrm{m}$; Agilent Technologies) at $40^{\circ} \mathrm{C}$. The mobile phase consisted of 10 $\mathrm{mmol} / \mathrm{L}$ aqueous ammonium acetate solution and acetonitrile at a flow rate of $0.2 \mathrm{~mL} / \mathrm{min}$. The gradient elution program was set as follows: $0-1 \mathrm{~min}$ hold on $10 \%$ acetonitrile, 1-20 min linear increase on $10-80 \%$ acetonitrile, and $20-35$ min hold on $80 \%$ acetonitrile. The mass spectrometer was operated in selected reaction monitoring

\footnotetext{
${ }^{* 1}$ Notification 14729 (Apr. 1, 2008) Food Safety and Consumer Affairs Bureau, Ministry of Agriculture, Forestry and Fisheries, Japan.
} 
Table 1. LC-MS/MS conditions, LOD and LOQ

\begin{tabular}{|c|c|c|c|c|c|c|c|c|}
\hline \multirow{2}{*}{ Mycotoxin } & \multirow{2}{*}{$\begin{array}{c}\text { Precursor ion } \\
(m / z)\end{array}$} & \multicolumn{2}{|c|}{ Product ion $(m / z)$} & \multirow{2}{*}{$\begin{array}{c}\text { Cone voltage } \\
\text { (V) }\end{array}$} & \multicolumn{2}{|c|}{ Collision energy $(\mathrm{eV})$} & \multirow{2}{*}{$\begin{array}{c}\mathrm{LOD} \\
(\mu \mathrm{g} / \mathrm{kg})\end{array}$} & \multirow{2}{*}{$\begin{array}{c}\mathrm{LOQ} \\
(\mu \mathrm{g} / \mathrm{kg})\end{array}$} \\
\hline & & Target & Qualifier & & Target & Qualifier & & \\
\hline ZEN & 317 & 175 & 131 & 52 & 24 & 32 & 0.3 & 1 \\
\hline $\mathrm{ZAN}$ & 319 & 205 & 275 & 62 & 26 & 24 & 2 & 4 \\
\hline$\alpha$ - and $\beta$-ZEL & 319 & 160 & 275 & 62 & 28 & 24 & 3 & 8 \\
\hline$\alpha$ - and $\beta$-ZAL & 321 & 277 & 303 & 56 & 24 & 24 & 2 & 4 \\
\hline
\end{tabular}

Table 2. Production of ZEN and the 5 ZRCs during incubation of F. semitectum on sorghum or rice

\begin{tabular}{|c|c|c|c|c|c|c|c|}
\hline \multirow{2}{*}{ Substrate } & \multirow{2}{*}{ Fungus No. } & \multirow{2}{*}{ ZEN (mg/kg) } & \multicolumn{5}{|c|}{ Ratio relative to ZEN (\%) } \\
\hline & & & ZAN & $\alpha$-ZEL & $\beta$-ZEL & $\alpha$-ZAL & $\beta-\mathrm{ZAL}$ \\
\hline \multirow[t]{8}{*}{ Sorghum $^{\text {a) }}$} & M-4-2 & 19400 & 0.80 & 0.05 & 0.52 & $<0.01$ & 0.01 \\
\hline & M-8-1 & 10600 & 1.1 & 0.02 & 0.11 & $<0.01$ & $<0.01$ \\
\hline & M-8-3 & 1530 & 1.6 & 0.06 & 0.60 & $<0.01$ & 0.03 \\
\hline & M-8-4 & 6850 & 1.2 & 0.58 & 24 & 0.07 & 0.59 \\
\hline & M-8-5 & 2170 & 1.5 & 0.04 & 0.22 & $<0.01$ & 0.01 \\
\hline & M-158-1 & 1640 & 2.2 & 0.17 & 2.8 & 0.01 & 0.12 \\
\hline & M-158-4 & 9290 & 1.2 & 0.02 & 0.15 & $<0.01$ & 0.01 \\
\hline & M-480-3 & 4560 & 1.0 & 0.15 & 3.2 & 0.01 & 0.09 \\
\hline \multirow[t]{2}{*}{ Rice $^{\text {b) }}$} & M-8-1 & 83.5 & 2.4 & 1.9 & 3.6 & 0.06 & 0.22 \\
\hline & M-158-4 & 124 & 1.5 & 0.48 & 4.1 & 0.01 & 0.14 \\
\hline
\end{tabular}

a) One substrate per strain was incubated at $25^{\circ} \mathrm{C}$ for 14 days.

b) Five substrates per strain were incubated at $25^{\circ} \mathrm{C}$ for 14 days and the mean value is shown.

(SRM) mode at negative polarity. The capillary voltage was set to $0.6 \mathrm{kV}$, and the source block and desolvation gas temperatures were set to 150 and $400^{\circ} \mathrm{C}$, respectively. The desolvation and cone gas were nitrogen at 800 and $50 \mathrm{~L} / \mathrm{h}$, respectively, and the collision gas used was argon. The monitoring ions, cone voltages, and collision energies for ZEN and the 5 ZRCs are shown in Table 1. Stock solutions were mixed to prepare standard solutions for calibration. The mixed solution was diluted in acetonitrile-water $(21: 4)$ to different concentrations and then mixed with the same amount of water-acetic acid $(100: 1)$ for LC-MS/MS. The peak signal areas of ZEN and the 5 ZRCs were plotted against those of standard solutions of different concentrations, and the concentration of ZEN or the 5 ZRCs in the sample solution was calculated from the calibration curve. The limit of detection (LOD) and the limit of quantification (LOQ) in the sample are shown in Table 1.

\section{Results and Discussion}

The amount of ZEN and the formation ratio of each of the $5 \mathrm{ZRCs}$ to ZEN produced by incubation of $F$. semitectum on sorghum or rice are shown in Table 2. F. semitectum is the primary causative fungus of ZEN contamination on sorghum ${ }^{20)}$. Therefore, sorghum was used as the primary substrate, and rice was employed as a reference substrate for comparison. The results revealed that ZAN and $\beta$-ZEL were dominant among the ZRCs on both substrates. ZAN is sometimes used as an internal standard for the analysis of ZEN and other mycotoxins, including trichothecene and aflatoxins ${ }^{8,21-23)}$. Our study revealed that ZAN was formed dominantly among the $5 \mathrm{ZRCs}$, suggesting that it may be unsuitable as an internal standard. The amount of $\beta$-ZEL relative to ZEN produced on the sorghum substrate was the highest among the $5 \mathrm{ZRCs}$ at $24 \%$. The ratio of $\alpha$-ZAL to ZEN was consistently the lowest of the 5 ZRCs in all strains and substrates, with its highest value being $0.07 \%$. Some strains of $F$. semitectum studied did not produce ZEN or the 5 ZRCs, although previous studies have reported production of the 5 ZRCs upon incubation of Fusarium fungi on medium. Bottalico et al. examined the production of $\alpha$ and $\beta$-ZEL by Fusarium species associated with corn stalk $\operatorname{rot}^{11)}$. In that study, incubation of Fusarium fungi on potato glucose agar resulted in the production of $\alpha$ and $\beta$-ZEL. Their formation ratios by $F$. culmorum were $0.03-0.65 \%$ and $0.02-0.43 \%$ relative to $\mathrm{ZEN}$, respectively. $F$. equiseti gave ratios of $3.3-4.8 \%$ and $13-29 \%$, respectively. These findings are similar to those observed in our study, although different Fusarium fungi were used. Additionally, Richardson et al. also investigated the production of $\alpha$ - or $\beta$-ZEL and $\alpha$ - or $\beta$-ZAL by incubation of Fusarium fungi isolated from finished feeds and forage plants ${ }^{12)}$. When $F$. graminearum and other fungi were grown on rice culture, $\alpha$-ZEL, $\beta$-ZEL, $\alpha$-ZAL, and $\beta$-ZAL were produced at ratios of $1.3-20 \%, 0.34-110 \%$, $0-13 \%$, and $0-14 \%$ relative to $\mathrm{ZEN}$, respectively. Although $\beta$-ZEL was not dominant among the $5 \mathrm{ZRCs}$ in that study, the ratios of the $5 \mathrm{ZRCs}$ to ZEN were similar to those observed in our study. Furthermore, we observed higher levels of $\alpha$ - and $\beta$-ZEL than $\alpha$ - and $\beta$-ZAL, which is also consistent with their results. In relation to the estrogenic activity of ZEN, the activities of $5 \mathrm{ZRCs}$ cannot be ignore, because $\alpha$-ZEL and ZAN were pro- 
duced in artificial culture and they both possess higher estrogenic activities than ZEN (Fig. 1).

To examine the time courses of production of ZEN and the 5 ZRCs in culture of the M-8-4 F. semitectum strain (Table 2), the fungus was grown on sorghum for 3 to 15 days. The concentration changes of ZEN and the $5 \mathrm{ZRCs}$ are shown in Fig. 2. All compounds increased until 9 days after the start of the incubation period, and then further increased slightly or stayed constant from 9 to 15 days. Narita et al. examined the production of ZEN, $\alpha$-ZEL, and $\beta$-ZEL during incubation of $F$. graminearum on potato dextrose agar medium ${ }^{24)}$, and reported that the production profiles of $\alpha$-ZEL and $\beta$-ZEL were similar to that of ZEN with three of the four strains tested, but a different production profile was observed with the other strain. Our results also indicated that the production of the $5 \mathrm{ZRCs}$ increased in a manner similar to ZEN.

To examine the production of the $5 \mathrm{ZRCs}$ in more detail, a time course analysis of the production ratios of the $5 \mathrm{ZRCs}$ relative to ZEN was performed (Fig. 3). The ratios of $\beta$-ZEL, $\alpha$-ZAL, and $\beta$-ZAL decreased from 3 to 15 days. However, the ratio of $\alpha$-ZEL reached its maxi-

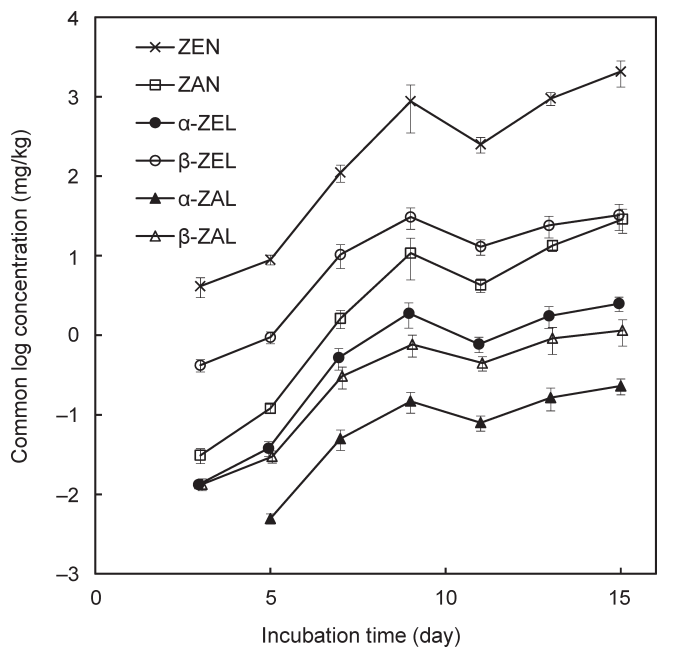

Fig. 2. Time course analysis of the production of ZEN and the $5 \mathrm{ZRCs}$

The M-8-4 strain of $F$. semitectum was incubated on the sorghum substrate. The data represent the mean of triplicate (3 to 11 days) or quintuplicate (13 and 15 days) values \pm the standard error of the mean. mum at 7 days, and thereafter decreased similarly. ZAN increased until 5 days, after which its production remained constant. These results suggest that the contribution of the five ZEN-related compounds to the estrogenic activity of ZEN will decrease over time. Although the production of $\beta$-ZEL amounted to $24 \%$ relative to ZEN (Table 2), the time course analysis did not reveal a similar phenomenon (Fig. 3).

To observe the natural occurrence of the $5 \mathrm{ZRCs}$ in sorghum, we examined the levels of ZEN and the 5 ZRCs in five imported sorghums collected at a silo (Table 3). ZAN and $\alpha$ - and $\beta$-ZEL were detected. This result, like the in vitro incubation analysis, also brings into question the suitability of using ZAN as an internal standard. The ratios of ZAN and $\beta$-ZEL to ZEN, observed in naturally contaminated sorghum, were similar to those observed in culture of $F$. semitectum. However, the ratio of $\alpha$-ZEL to ZEN in sorghum was larger than that observed in culture. Of the $5 \mathrm{ZRCs}$, only $\alpha$-ZEL contamination levels in sorghum have been previously re-

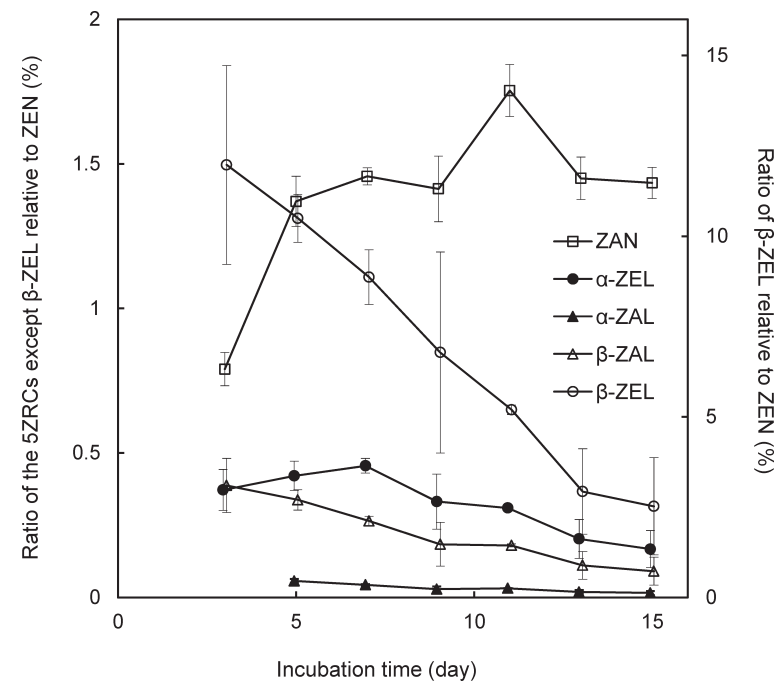

Fig. 3. Time course of the production ratios of the $5 \mathrm{ZRCs}$ relative to $\mathrm{ZEN}$

The M-8-4 strain of $F$. semitectum was incubated on a sorghum substrate. The scale for $\beta$-ZEL production ratio is on the right $Y$-axis and that of the other ZRCs is shown on the left $Y$-axis. The data represent the mean of triplicate (3 to 11 days) or quintuplicate (13 and 15 days) values \pm the standard error of the mean.

Table 3. ZEN and the $5 \mathrm{ZRCs}$ in naturally contaminated sorghum

\begin{tabular}{|c|c|c|c|c|c|c|}
\hline \multirow{2}{*}{ Sorghum No. } & \multirow{2}{*}{$\mathrm{ZEN}(\mu \mathrm{g} / \mathrm{kg})$} & \multicolumn{5}{|c|}{ Ratio relative to $\mathrm{ZEN}^{\mathrm{a})}(\%)$} \\
\hline & & $\mathrm{ZAN}$ & $\alpha-\mathrm{ZEL}$ & $\beta$-ZEL & $\alpha-\mathrm{ZAL}$ & $\beta$-ZAL \\
\hline $1-128$ & 313 & 2.7 & $\mathrm{ND}$ & 3.1 & ND & ND \\
\hline $2-35$ & 303 & 1.4 & 1.7 & $\mathrm{ND}$ & ND & ND \\
\hline $3-116$ & 178 & ND & 2.4 & 2.4 & ND & ND \\
\hline $3-170$ & 194 & 5.5 & 3.5 & 4.5 & ND & ND \\
\hline $3-253$ & 211 & ND & 2.5 & 3.0 & ND & ND \\
\hline
\end{tabular}

\footnotetext{
a) Concentrations of LOD or more were used in the calculation.
} 
ported $^{25)}$. That study demonstrated that, similar to our findings, $\alpha$-ZEL was detected in two of five sorghums at ratios relative to ZEN of 5.3 and $2.8 \%$. Studies investigating the contamination of the 5 ZRCs in other crops $^{11,13)-17)}$ show that the levels of the 5 ZRCs are lower than that of ZEN, and ZAL was lower than the other ZRCs, as also observed in our study. It is difficult to reconstruct the in situ contamination ratio precisely from the in vitro incubation analysis, but in vitro analysis might be helpful as an indicator of possible contamination.

\section{References}

1) Kuiper-Goodman, T., Scott, P. M., Watanabe, H. Risk assessment of the mycotoxin zearalenone. Regul. Toxicol. Pharmacol., 7, 253-306 (1987).

2) Tanaka, T., Hasegawa, A., Yamamoto, S., Lee, U-S., Sugiura, Y., Ueno, Y. Worldwide contamination of cereals by the Fusarium mycotoxins nivalenol, deoxynivalenol, and zearalenone. 1. Survey of 19 countries. J. Agric. Food Chem., 36, 979-983 (1988)

3) Zinedine, A., Soriano, J. M., Moltó, J. C., Mañes, J. Review on the toxicity, occurrence, metabolism, detoxification, regulations and intake of zearalenone: An oestrogenic mycotoxin. Food Chem. Toxicol., 45, 1-18 (2007).

4) Hagler, W. M., Mirocha, C. J., Pathre, S. V., Behrens, J. C. Identification of the naturally occurring isomer of zearalenol produced by Fusarium roseum 'Gibbosum' in rice culture. Appl. Environ. Microbiol., 37, 849-853 (1979).

5) Le Guevel, R., Pakdel, F. Assessment of oestrogenic potency of chemicals used as growth promoter by in-vitro methods. Hum. Reprod., 16, 1030-1036 (2001).

6) Shier, W. T., Shier, A. C., Xie, W., Mirocha, C. J. Structure-activity relationships for human estrogenic activity in zearalenone mycotoxins. Toxicon, 39, 1435-1438 (2001).

7) Zöllner, P., Jodlbauer, J., Kleinova, M., Kahlbacher, H., Kuhn, T., Hochsteiner, W., Lindner, W. Concentration levels of zearalenone and its metabolites in urine, muscle tissue, and liver samples of pigs fed with mycotoxincontaminated oats. J. Agric. Food Chem., 50, 2494-2501 (2002).

8) Zöllner, P., Berner, D., Jodlbauer, J., Lindner, W. Determination of zearalenone and its metabolites $\alpha$ - and $\beta$-zearalenol in beer samples by high-performance liquid chromatography-tandem mass spectrometry. J. Chromatogr. B, 738, 233-241 (2000).

9) Nakajima, M. Studies on mycotoxin analysis using immunoaffinity column. Mycotoxins, 53, 43-52 (2003).

10) Scott, P. M. Mycotoxins transmitted into beer from contaminated grains during brewing. J. AOAC Int., 79, 875882 (1996).

11) Bottalico, A., Visconti, A., Logrieco, A., Solfrizzo, M., Mirocha, C. J. Occurrence of zearalenols (diastereomeric mixture) in corn stalk rot and their production by associated Fusarium species. Appl. Environ. Microbiol., 49, 547-551 (1985)

12) Richardson, K. E., Hagler, W. M., Mirocha, C. J. Pro- duction of zearalenone, $\alpha$ - and $\beta$-zearalenol, and $\alpha$ - and $\beta$ zearalanol by Fusarium spp. in rice culture. J. Agric. Food Chem., 33, 862-866 (1985).

13) Mirocha, C. J., Schauerhamer, B., Christensen, C. M., Niku-Paavola, M. L., Nummi, M. Incidence of zearalenol (Fusarium mycotoxin) in animal feed. Appl. Environ. Microbiol., 38, 749-750 (1979).

14) Cavaliere, C., D’Ascenzo, G., Foglia, P., Pastorini, E., Samperi, R., Laganà, A. Determination of type B trichothecenes and macrocyclic lactone mycotoxins in field contaminated maize. Food Chem., 92, 559-568 (2005).

15) Schollenberger, M., Müller, H-M., Rüfle, M., Suchy, S., Plank, S., Drochner, W. Natural occurrence of $16 \mathrm{Fu}$ sarium toxins in grains and feedstuffs of plant origin from Germany. Mycopathologia, 161, 43-52 (2006).

16) Jin, P. G., Han, Z., Cai, Z. X., Wu, Y. J., Ren, Y. P. Simultaneous determination of 10 mycotoxins in grain by ultra-high-performance liquid chromatography-tandem mass spectrometry using ${ }^{13} \mathrm{C}_{15}$-deoxynivalenol as internal standard. Food Addit. Contam., 27, 1701-1713 (2010).

17) De Boevre, M., Jacxsens, L., Lachat, C., Eeckhout, M., Di Mavungu, J. D., Audenaert, K., Maene, P., Haesaert, G., Kolsteren, P., De Meulenaer, B., De Saeger, S. Human exposure to mycotoxins and their masked forms through cereal-based foods in Belgium. Toxicol. Lett., 218, 281292 (2013).

18) JECFA, Zearalenone, Safety evaluation of certain food additives and contaminants, WHO food additives series 44, IPCS-International Programme on Chemical Safety, WHO, Geneva (2000).

19) Codex Alimentarius Commission, Discussion paper on mycotoxins in sorghum. Joint FAO/WHO Food Standards Programme, Codex Committee on Contaminants in Foods 5th session held in Hague, the Netherlands on 2125 March 2011 (2011).

20) Aoyama, K., Ishikuro, E., Nishiwaki, M., Ichinoe, M. Zearalenone contamination and the causative fungi in sorghum. J. Food Hyg. Soc. Jpn., 50, 47-51 (2009).

21) Zöllner, P., Jodlbauer, J., Lindner, W. Determination of zearalenone in grains by high-performance liquid chromatography-tandem mass spectrometry after solid-phase extraction with RP-18 columns or immunoaffinity columns. J. Chromatogr. A, 858, 167-174 (1999).

22) Biselli, S., Hummert, C. Development of a multicomponent method for Fusarium toxins using LC-MS/MS and its application during a survey for the content of T-2 toxin and deoxynivalenol in various feed and food samples. Food Addit. Contam., 22, 752-760 (2005).

23) Ren, Y., Zhang, Y., Shao, S., Cai, Z., Feng, L., Pan, H., Wang, Z. Simultaneous determination of multi-component mycotoxin contaminants in foods and feeds by ultraperformance liquid chromatography tandem mass spectrometry. J. Chromatogr. A, 1143, 48-64 (2007).

24) Narita, N., Suzuki, M., Tanaka, T., Ikebuchi, H., Ichinoe, M. Production of zearalenone and zearalenol by Fusarium graminearum. Mycotoxins, 40, 56-59 (1994).

25) Bagneris, R. W., Gaul, J. A., Ware, G. M. Liquid chromatographic determination of zearalenone and zearalenol in animal feeds and grains, using fluorescence detection. J. Assoc. Off. Anal. Chem., 69, 894-898 (1986). 